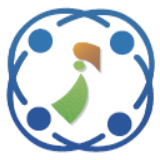

\title{
Adaptive Noise Detection Using Texture Feature Extraction and Random Forest Classification
}

\author{
Arvind Kumar Ganesh ${ }^{1 *} \quad$ Ashok Kusagur $^{2}$ \\ ${ }^{1}$ Department of Telecommunication Engineering, \\ Ramaiah Institute of Technology, India \\ ${ }^{2}$ Department of Electrical \& Electronics Engineering, \\ University B.D.T. College of Engineering, India \\ * Corresponding author's Email: arvind@ msrit.edu
}

\begin{abstract}
Image noise detection is one of the most challenging aspect in the field of image processing and analysis. Generally, the noise affects the information of image, especially when the noise level is too high. To avoid this kind of issue, image denoising schemes is developed for several applications: medical image analysis, pattern recognition, biometric authentication, etc. to reduce the noise level in the image. This experimental research concentrates on two objectives i.e. identify the noise in the corrupted image and suggest the denoising algorithm for the respective noise. To perform this operation, a new methodology is developed, initially, noise subtraction is carried-out to separate the noisy image and higher frequency image components from the digital images. Then, feature extraction is carried-out on noisy image using texture features like homogeneity, contrast, correlation, energy and two Dimension-Discrete Wavelet Transform (DWT) for extracting the optimal feature values. The obtained feature vectors are given as the input for multi-objective classifier: Random Forest (RF) in order to classify the type of noise. Experimental outcome showed that the proposed technique improved the noise prediction accuracy up to 5-20\% compared to the existing methods: Neural Network (NN), Naïve Bayes (NB) classifier, K-Nearest Neighbor (KNN) and Convolutional Neural Network (CNN) by means of accuracy, sensitivity and specificity.
\end{abstract}

Keywords: K-nearest neighbor, Naïve Bayes classifier, Neural network, Random forest, Wavelet transform.

\section{Introduction}

The digital images play an exceptional role in several applications like resonance imaging, computed tomography, medical images, satellite television, etc. and also in the fields of engineering science (astronomy and geographical systems) and medical analysis $[1,2]$. Generally, the digital images are susceptible to a variety of noises that affect the image quality [3]. To overcome this concern, image denoising methodologies are developed to restore the information of the original image by eliminating the unwanted noise [4]. There are various denoising algorithms available to eliminate the noise from digital images. Whereas, the conventional denoising methodologies are designed by considering the parameters like artifacts and noise [5]. The filtering denoising methodologies have two steps: noise reduction filter adjusts the pixels and classifies as noise-corrupted and the impulse detector classifies the image pixels as noise-free or noise-corrupted [6]. Image denoising application remains a challenging area for researchers, because the noise leads to blur the digital images and also causes the artifacts $[7,8]$.

Most of the existing methods cannot judge whether the corrupted image is affected by which type of noise in advance [9], [10]. So, it is significant to construct a detector in digital images before performing denoising algorithms like discrete cosine transform [11], digital image correlation [12], decision based filtering [13], etc. In order to deal with the above problems, this paper presented a method to detect and remove the noises (Gaussian and impulse) from the digital images. The proposed 
method developed a new detector for corrupted images using two dimensional-DWT scheme along with RF classifier and texture features like homogeneity, contrast, correlation and energy. The developed method processes the noisy images and analysed the images, whether the noise is Gaussian or impulse noise. The proposed methodology analysis the digital images based on the texture features and two dimensional-DWT for extracting the optimal feature values. These extracted features are given as an input for RF classifier in order to predict the types of noises. The proposed technique is experimented and its performance is estimated using three performance evaluation measures like specificity, sensitivity and accuracy of different test images.

This paper is composed as follows. Section 2 surveys several recent papers on noise detection related strategies. In section 3 , texture feature extraction methods are presented with two dimensional-DWT and multi objective classification technique (RF). Section 4 represents the comparative analysis of existing and proposed strategy. The conclusion is made in Section 5.

\section{Literature review}

Several researchers suggested many techniques in the field of noise prediction. In this scenario, a brief evaluation of some important contributions to the existing literatures presented below.

P. Fu, C. Li, W. Cai, and Q. Sun, [14] proposed a new cohesive super-pixel methodology for decomposing a noisy image into patches to increase the homogeneity. In addition, a new pixel based similarity measure was developed to make the cohesive super-pixel methodology more robust to noise. Then, combined filter based noise level estimation and histogram based homogeneous super-pixel selection helps to determine the noise level accurately in the images. Extensive experiments were performed on the "Fish" image in the BSD database for demonstrating the effectiveness of the proposed method. The disadvantage of cohesive super-pixel methodology was more complex to find the projection of free space. Free space can able to provide better discriminant ability in feature extraction.

J. Constantin, A. Bigand, I. Constantin, and D. Hamad, [15] illustrated a relevant model: Fast Relevance Vector Machine (FRVM) for highlighting the perceptual noise in an image. The proposed model was utilized in many progressive stochastic global illumination approach for identifying the image visual convergence threshold.
Extensive experiments were conducted and the effectiveness of the proposed technique was related to the existing method: Support Vector Machine (SVM). Experimental outcome confirmed that the proposed technique out-performed the existing scheme in terms of mean square error. Though, FRVM was highly affected by the number of samples in the training set, when the number of samples was low that affects the prediction rate.

K. Ray, [16] presented a new unsupervised algorithm for both edge and noise detection using canny edge detection scheme. This technique was computationally cheap and simple to attain nontrivial outcome. In addition, the proposed approach generated the information about the severity and the content of the noise in an image. The developed methodology utilized fast edge detector for generating edge mask and also to optimize the details of proposed estimator. The experiments carried on publicly available digital images like Lena image and blooming flower image in order to validate its accuracy, robustness and speed. While performing with semi supervised methodologies, the semantic gap was maximized between the feature values, which leads to poor detection rate.

C. Catal, O. Alan, and K. Balkan, [17] developed an appropriate noise detection scheme based on software metric threshold values. The respective threshold values were achieved from the receiver operating characteristic analysis. In this literature, the experiment was carried on five NASA databases and also constructed a detail about Naïve Bayes based software fault detection models both after and before employing the proposed noise prediction methodology. Experimental outcome showed that the proposed noise prediction methodology was very effective to detect the class noise using Naive Bayes algorithm. In some cases, the training data were dependent evaluation or manual adjustment, which was needed to be automated.

X. Tian, and E. Samei, [18] proposed a devise methodology for measuring the quantum noise in CT images and also develop an approach for detecting the noise with higher accuracy. This literature consists of 83 clinical image datasets at two dose levels. The quantum noise in the CT images was estimated by filtering out the edges and subtracting the sequential slices. Then, the noise was measured in the resultant uniform area. Validated outcome confirmed that the proposed technique was very effective related to the existing schemes. In this research, it was so difficult to compare the noises, due to lower level alignment.

J. Joseph, and R. Subban, [19] presented a new denoising methodology, which was based on super- 
pixel clustering and dictionary learning process. Initially, super-pixel clustering approach groups the similar pixels together to form a super-pixel. Then, the super-pixels and sparse coefficients were computed by using steepest descent orthogonal matching pursuit methodology. The dictionary updating process was achieved by employing Discriminative K-Singular Value Decomposition (DK-SVD). The dictionary was trained by DK-SVD algorithm, as the discriminative capability of the algorithm was greater. The proposed methodology shows promising results, compared to the existing techniques. In this research paper, the time consumption of the proposed approach was slightly maximum than the comparative techniques.

To overcome the above mentioned drawbacks, an unsupervised algorithm (texture feature extraction with multi objective classifier: RF) is implemented to increase the performance of noise prediction.

\section{Proposed methodology}

The proposed noise detection system consists of four stages: image acquisition, noise subtraction, feature extraction and classification. The working process of the proposed noise detection system represented in the Fig. 1. A brief description about the proposed methodology is given below.

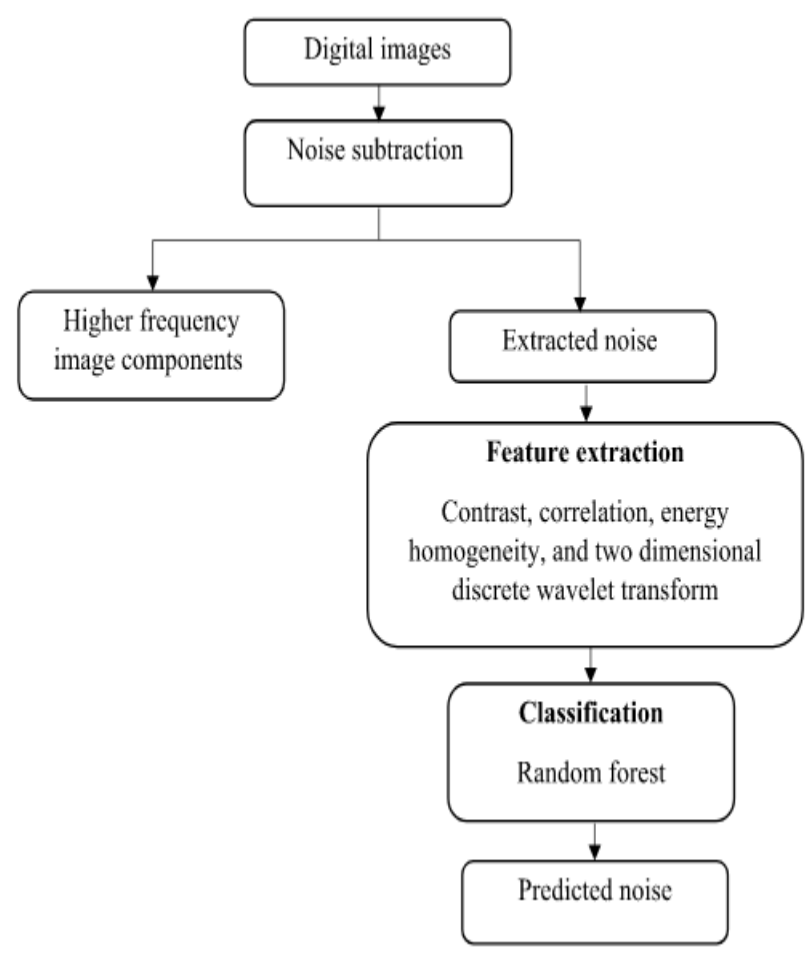

Figure. 1 working procedure of the proposed methodology

\subsection{Image acquisition}

In the initial stage of noise detection system, several standard digital images such as Lena, pepper, baboon, boat, Wang, MNIST database images, etc. are considered. It is necessary to select appropriate image, if the image is not acquired satisfactorily then the intended operations may not be achievable. After acquiring the digital images, noise subtraction is performed in order to separate the noisy image and higher frequency image components from the digital images. Noise subtraction is also known as noise detection, it is a technique in the fields of computer vision and image processing, wherein the image noise is extracted for further processing (feature extraction).

\subsection{Feature extraction}

In this research methodology, the feature extraction is carried-out on the separated noisy image. Generally, feature extraction is defined as the action of mapping the digital image from imagespace to the feature-space. In this scenario, the high level features named as Gray-Level Co-occurrence Matrix (GLCM) and two dimensional-DWT are employed for extracting the features of noisy image. GLCM is the most known texture analysis method that estimates image properties associated with second order statistics. Here, four GLCM texture features are considered: homogeneity, energy, correlation and contrast. The brief description about GLCM features and two dimensional-DWT are detailed below.

\subsubsection{Homogeneity}

Homogeneity determines the closeness of distribution elements in the gray level matrix. The distribution parameter is high compared to the evaluated methods. To quantitatively characterize the homogeneous texture regions for similarity, the local spatial statistics of the texture is calculated using scale and orientation selective of Gabor filtering. The digital images are divided into a set of homogeneous texture regions, then the texture features are compared to the indexed image region. In GLCM, homogeneity calculates four directions (i.e. $\Theta=0 \circ, 45^{\circ}, 90^{\circ}$ or $135^{\circ}$ ) with a feature vector size of four. Homogeneity delivers high accuracy of detection in the defected areas, which are determined by a weak variation in grey level. The general formula to determine the homogeneity of a digital image is given in the Eq. (1). 


$$
\text { Homogeneity }=\sum_{i=0}^{n-1} \sum_{j=0}^{n-1} \frac{P_{i j}}{1+(i-j)^{2}}
$$

Where, $P_{i j}$ is stated as the normalized cooccurrence matrix, $n$ is denoted as number of gray levels of digital image, $i$ and $j$ are represented as the intensities between 0 and number of levels -1 .

\subsubsection{Energy}

Energy evaluates the uniformity of normalized pixel pair distributions and the number of repeated pairs. Energy has a normalized pixel range with the highest value of one. If high energy value occurs, then the gray level distribution has a periodic form. Energy helps to reflect the depth and smoothness of the digital image texture structure. The general formula to determine the energy of a digital image is given in the Eq. (2).

$$
\text { Energy }=\sum_{i=0}^{n-1} \sum_{j=0}^{n-1}-\ln \left(P_{i j}\right) P_{i j}
$$

Where, $P_{i j}$ is represented as the normalized cooccurrence matrix and $n$ is stated as the number of gray levels of digital image

\subsubsection{Correlation}

Correlation evaluates the linear dependency of neighbouring grey level pixels. Digital image correlation is an optical technique that utilizes accurate two-dimensional and three dimensional measurements of digital images. This is often utilized to evaluate the strain, displacement, deformation, and optical flow in a digital image. Correlation widely applied in the field of image denoising, which helps to detect the disorders in the texture of an image. The general formula to determine the correlation of a digital image is given in the Eq. (3).

$$
\text { Correlation }=\sum_{i=0}^{n-1} \sum_{j=0}^{n-1} P_{i j} \frac{(i-\mu)(j-\mu)}{\sigma^{2}}
$$

Where, $\mu$ is represented as the mean, $\sigma$ is denoted as the standard deviation, $n$ is represented as number of gray levels of digital image and $P_{i j}$ is denoted as the normalized co-occurrence matrix

\subsubsection{Contrast}

Contrast determines the spatial frequency of digital images and also it evaluates the difference between the lowest and highest values of a contiguous set of pixels. It calculates the volume of local variations present in the digital image. This statistic reduces the complexity of the digital images. The general formula to determine the contrast of a digital image is given in the Eq. (4).

$$
\text { Contrast }=\sum_{i=0}^{n-1} \sum_{j=0}^{n-1} P_{i j}(i-j)^{2}
$$

Where, $P(i, j)$ represented as the pixel value of the position $(i, j)$ of the digital images and normalized co-occurrence matrix is denoted as $P_{i j}$.

\subsubsection{Two dimensional discrete wavelet transform}

DWT is an efficient tool for image processing applications and it is adopted in several emerging standards. The growing "success" of DWT in noise detection is due to multi-resolution processing capability. The DWT have properties like good compression of energy, no redundancy and very low computation. Also, DWT is utilized as a fuzzy denoising approach, which delivers good directional selectivity and shift able sub-bands. In the multiresolution technique, the underlying texture of digital images are analysed by zooming in and out process. Initially, DWT decomposes the digital image into a number of sub-images in dissimilar resolution levels for preserving the low and high frequency information. This property leads the wavelet to extract better texture information from the digital images. The square integrable function $f(x)$ and its wavelet transform is determined as the inner product of $f$ and a real valued function $\Psi(x)$ is given in the Eq. (5).

$$
w[f(s, \tau)]=\left(f, \Psi_{s, t}^{k}\right)=\int_{-\infty}^{\infty} f(x) \Psi_{s, t}^{k}(x) d x
$$

Where, $\Psi_{s, t}^{k}(x)=\left(\frac{1}{\sqrt{s} \Psi_{s, t}^{k}(x-\tau)} / s\right)$ is represented as wavelet family, $s \in Z, \tau$ and $k \in\{h, v, d\}$ are denoted as scale (resolution level), translation and orientation parameters. The orientation parameters $h, v$, and $d$ are represented as horizontal, vertical and diagonal directions. Now, the dyadic wavelet decomposition is achieved, when $s=2^{j}$ and $\tau=2^{j}, n, j, n \in Z$. The wavelet function $\Psi(x)$ and the scaling function $\varphi(x)$ are used to construct the wavelet and scaling families using the Eqs. (6) and (7).

$$
\begin{aligned}
& \Psi_{j, n}^{k}(x)=\frac{1}{\sqrt{2^{j}}} \Psi^{k}\left(\frac{x-2^{j} . n}{2^{j}}\right) \\
& \varphi_{j, n}^{k}(x)=\frac{1}{\sqrt{2^{j}}} \varphi^{k}\left(\frac{x-2^{j} . n}{2^{j}}\right)
\end{aligned}
$$




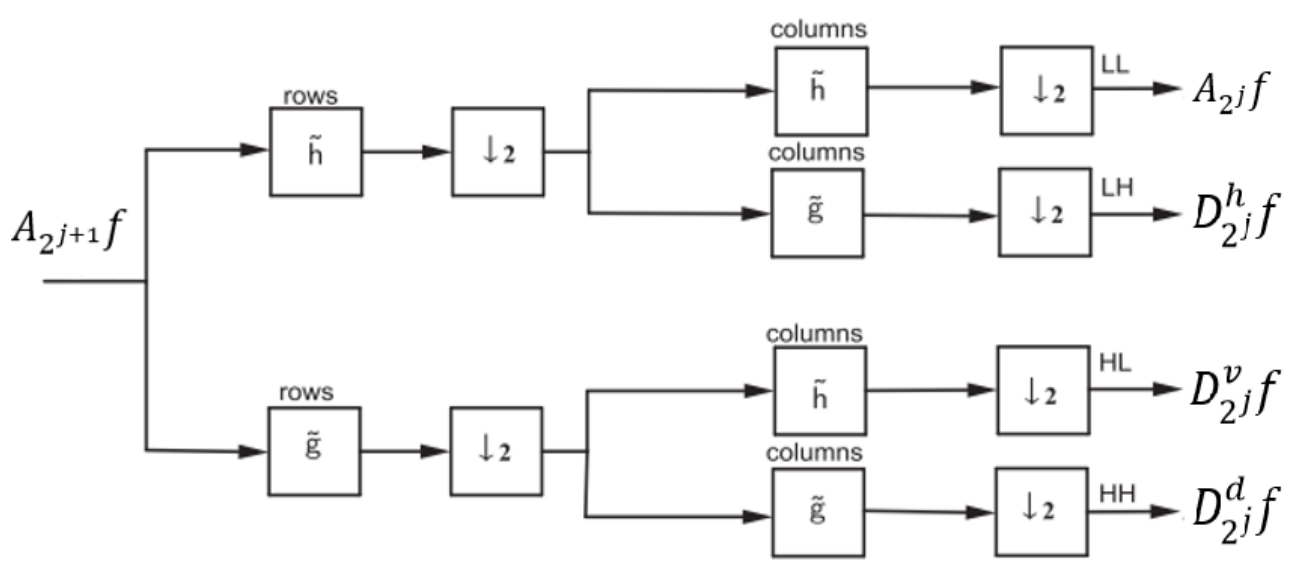

Figure.2 Wavelet decomposition using analysis filter banks

These are orthonormal basis of sub-spaces and related to resolution $2^{j}$. The wavelet atoms are determined by three mother atoms: $\Psi^{h}, \Psi^{v}$ and $\Psi^{d}$. These oriented mother atoms are computed as the tensor product of one dimensional $\Psi(x)$ and $\varphi(x)$ given in the Eqs. (8) and (9).

$$
\begin{aligned}
& \varphi(x)=\varphi\left(x_{1}\right) \varphi\left(x_{2}\right), \Psi^{h}(x)=\Psi\left(x_{1}\right) \Psi\left(x_{2}\right) \\
& \Psi^{v}(x)=\varphi\left(x_{1}\right) \Psi\left(x_{2}\right), \Psi^{d}(x)=\Psi\left(x_{1}\right) \varphi\left(x_{2}\right)
\end{aligned}
$$

A two dimensional DWT is employed by using the combination of down-samplers and digital filter banks. The digital filter banks consist of high-pass $(g)$ and low-pass $(h)$ filters. In the configuration of DWT structure, the number of banks is set as per the desired resolution. As the image is a two dimensional, separable wavelet functions are computed using DWT. The columns and rows of the image are separately undergone through the one dimensional wavelet transform to establish the two dimensional DWT. As shown in the Fig. 2, the original digital images $A_{2^{j+1}} f$ at resolution $2^{j+1}$ is decomposed into four sub-band images in the frequency domain.

Among four sub-band images, three sub-band images $D_{2^{j}}^{h} f, D_{2^{j}}^{v} f, D_{2^{j}}^{d} f$ are the digital images at resolution $2^{j}$ in horizontal, vertical and diagonal directions respectively. The fourth one is the approximation image, $A_{2^{j}} f$ found at coarse resolution, so, the whole image $A_{2^{j+1}} f$ is represented in the Eq. (10).

$$
A_{2^{j+1}} f=D_{2^{j}}^{h} f+D_{2^{j}}^{v} f+D_{2^{j}}^{d} f+A_{2^{j}} f
$$

The decomposed sub-images are the representation of two dimensional orthogonal wavelet. Thus, the output of a wavelet decomposition of a digital image results into four orthogonal sub-band components like Low-Low (LL) band, Low-High (LH) band, High-Low (HL) band, and High-High $(\mathrm{HH})$ band, which correspond to sub-images $D_{2^{j}}^{h} f, D_{2^{j}}^{v} f, D_{2^{j}}^{d} f$ and $A_{2^{j}} f$.

\subsection{Classification using random forest}

The obtained feature value is given as the input for multi-objective classifier: RF classifier. It is one of the emerging classification methods with the advantage of limited usage of resources, while training the large dataset. RF is a non-parametric pattern classification approach and also one of the most extensively utilized un-supervised machine learning classification approach, which decreases the problem of probability density complexity. In RF classifier, each tree is observed as an individual classifier and the classification outcome is chosen by all the decision trees. To develop a RF classification approach, the growth rules of each tree are determined as follows.

If, $N$ is the number of training set, then randomly sample the $N$ data from the training set. $M$ is the dimension of input features. If $m(m<M)$, select the sub-features from the original feature vectors. Then, $m$ feature variables are chosen randomly out of the $M$ features and the best split on these $m$-dimensional features are utilized to split the node. Each tree keeps growing until all these training samples are totally separated without pruning. As revealed, the forest error rate depends on two aspects.

- The correlation between the two trees in the forest:

More correlation leads to bigger error rate and less correlation leads to smaller error rate. 
- Strength of each individual tree in the forest:

Increase of tree strength reduce the forest error rate.

\section{Result and discussion}

The proposed methodology was experimented using MATLAB (version 2017a) with 4 GB RAM, $3.0 \mathrm{GHz}$ Intel i3 processor and $500 \mathrm{~GB}$ hard disc. In order to determine the efficiency of the proposed algorithm, the proposed methodology performance was compared with existing methodologies: NN, $\mathrm{NB}, \mathrm{KNN}$, and CNN [20]. The proposed technique performance was compared by means of sensitivity, accuracy and specificity.

\subsection{Performance measure}

The relationship between the input and output variables of a system is understand by employing the suitable performance metrics like sensitivity and specificity. The general formula for calculating the sensitivity and specificity of noise detection is given in the Eqs. (12) and (13).

$$
\begin{aligned}
\text { Sensitivity }= & \frac{\text { NumberofTP }}{\begin{array}{c}
\text { NumberofTP }+ \text { NumberofFN } \\
\times 100
\end{array}} \\
\text { Specificity }= & \frac{\text { NumberofTN }}{\begin{array}{l}
\text { NumberofTN }+ \text { NumberofFP } \\
\times 100
\end{array}}
\end{aligned}
$$

In addition, accuracy is another suitable evaluation metric for finding the effectiveness of the noise detection system. The general formula of accuracy for determining the noise detection is given in the Eq. (14)

$$
\text { Accuracy }=\frac{T P+T N}{T P+T N+F P+F N} \times 100
$$

Where, $T P$ is denoted as true positive, $F P$ is denoted as false positive, $T N$ is stated as true negative and $F N$ is stated as false negative.

\subsection{Experimental analysis}

In this experimental analysis, several digital images (Lena, pepper, baboon, boat, Wang, MNIST database images, etc.) are utilized for comparing the performance evaluation of existing methods and the proposed scheme. Here, the performance evaluation is validated for random 20 digital images with $80 \%$ of training and $20 \%$ of testing. In Table 1, the performance of the proposed and existing methodologies is compared with $5 \%$ of noise. The accuracy value of existing methods: $\mathrm{KNN}, \mathrm{NN}$ and NB delivered $91 \%, 77.40 \%$ and $86.60 \%$ of accuracy and the proposed approach delivers $96.60 \%$ of accuracy. Similarly, the specificity value of existing methods delivers $91 \%, 66.72 \%$ and $86.60 \%$ of specificity and the proposed approach delivers $96.60 \%$ of specificity. In addition, the sensitivity value of existing methods delivered $97.75 \%$, $95.38 \%$ and $96.65 \%$ of sensitivity and the proposed approach delivers $99.15 \%$ of sensitivity. The graphical representation of performance evaluation of both existing and proposed methods are

\begin{tabular}{|c|c|c|c|c|c|c|c|c|c|c|c|c|}
\hline \multirow{2}{*}{$\begin{array}{c}\text { With-DWT (0.05) / } \\
(5 \%)\end{array}$} & \multicolumn{4}{|c|}{ Accuracy (\%) } & \multicolumn{4}{|c|}{ Specificity (\%) } & \multicolumn{4}{|c|}{ Sensitivity (\%) } \\
\hline & KNN & NN & $\mathbf{R F}$ & NB & KNN & NN & $\mathbf{R F}$ & NB & KNN & NN & $\mathbf{R F}$ & NB \\
\hline & 81.40 & 26.60 & 82.20 & 78.00 & 81.40 & 22.02 & 82.20 & 78.00 & 95.35 & 85.46 & 95.55 & 94.50 \\
\hline & 0 & 4 & 38.80 & & 3 & 39. & 0.0 & 38. & 4.60 & & $\underline{0}$ & 55 \\
\hline Energ & 85.40 & 28.80 & 86.00 & 67.60 & 85.40 & 27.91 & 86.00 & 67.60 & 96.35 & 2.84 & 96.50 & 91.90 \\
\hline & 9.60 & 8.6 & 79.20 & 65. & 79.60 & 27.2 & 79.20 & 65.2 & 94.90 & 6 & 94.80 & 91.30 \\
\hline & 76.80 & 25.60 & 72.00 & 66.4 & 76.80 & 24.43 & 72.00 & 66.40 & 94.20 & 82.37 & 93.00 & 91.60 \\
\hline $\begin{array}{r}\mathrm{Hyb} \\
\text { withou }\end{array}$ & 92.00 & 67.20 & 90.60 & 83.20 & 92.00 & 59.15 & 90.60 & 83.20 & 98.00 & 93.05 & 97.65 & 95.80 \\
\hline $\begin{array}{c}\text { Hybridization using } \\
\text { DWT }\end{array}$ & 91.00 & 77.40 & 96.60 & 86.60 & 91.00 & 66.72 & 96.60 & 86.60 & 97.75 & 95.38 & 99.15 & 96.65 \\
\hline
\end{tabular}
represented in the Figs. 3, 4, and 5.

Table 1. Performance evaluation of proposed and existing techniques with $5 \%$ of noise 


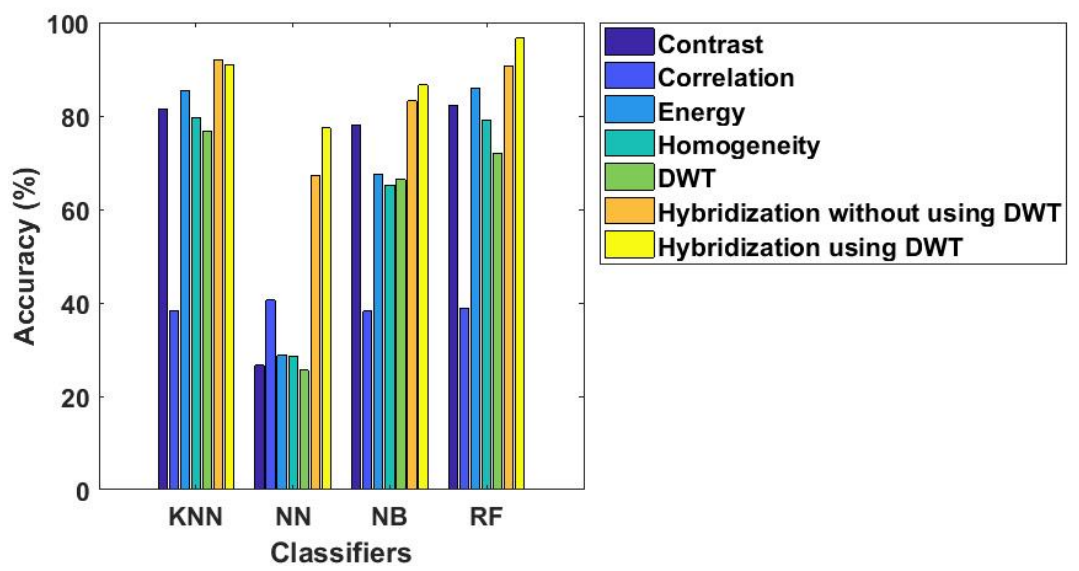

Figure 3 Accuracy comparison of proposed and existing techniques with 5\% of noise

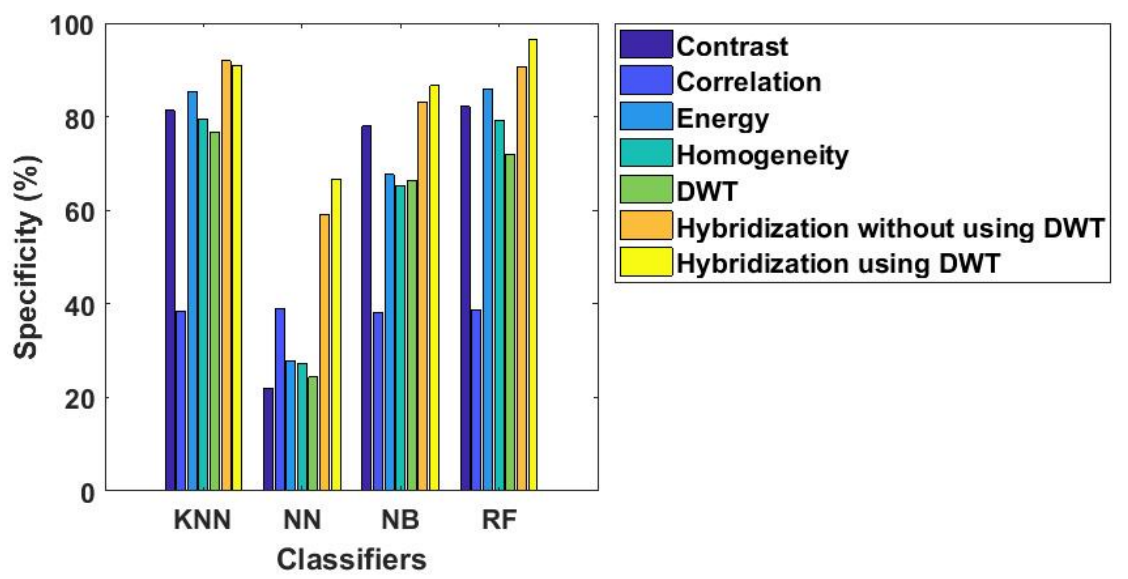

Figure.4 Specificity comparison of proposed and existing methods with 5\% of noise

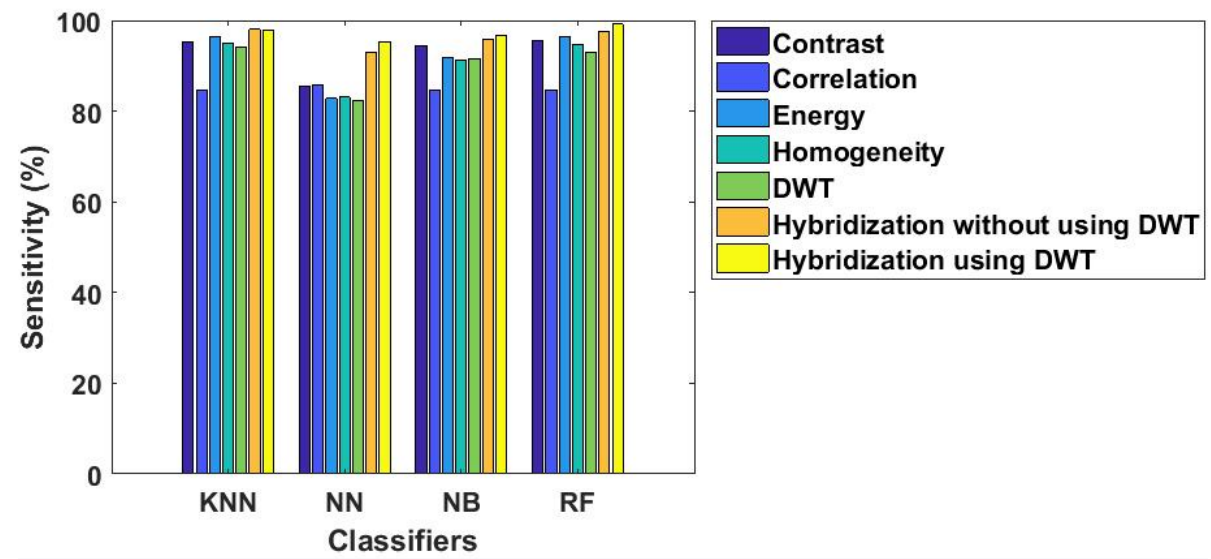

Figure.5 Sensitivity comparison of proposed and existing methods with $5 \%$ of noise

Table 2 evaluates the performance evaluation of proposed and existing methodologies with $80 \%$ of noise. Here, the accuracy value of existing classification methods: KNN, NN and NB delivered $86.20 \%, 77.80 \%$ and $88.20 \%$ of accuracy and the proposed approach delivered $96.40 \%$ of accuracy. Similarly, the specificity value of existing methods delivered $86.20 \%, 62.74 \%$ and $88.20 \%$ of specificity and the proposed approach delivered $96.40 \%$ of specificity. Furthermore, the sensitivity value of existing methods delivered $96.55 \%, 95.98 \%$ and $97.05 \%$ of sensitivity and the proposed approach delivered $99.10 \%$ of sensitivity. The graphical representation of performance evaluation of both existing and proposed methods are indicated in the Figs. 6, 7, and 8. 
Table 2. Performance evaluation of proposed and existing techniques with $80 \%$ of noise

\begin{tabular}{|c|c|c|c|c|c|c|c|c|c|c|c|c|}
\hline \multirow{2}{*}{$\begin{array}{c}\text { With-DWT } \\
(\mathbf{0 . 8}) /(80 \%)\end{array}$} & \multicolumn{4}{|c|}{ Accuracy (\%) } & \multicolumn{4}{c|}{ Specificity (\%) } & \multicolumn{4}{c|}{ Sensitivity (\%) } \\
\cline { 2 - 14 } & KNN & NN & RF & NB & KNN & NN & RF & NB & KNN & NN & RF & NB \\
\hline Contrast & 83.80 & 29.80 & 79.40 & 81.80 & 83.80 & 26.42 & 79.40 & 81.80 & 95.95 & 85.03 & 94.85 & 95.45 \\
\hline Correlation & 77.60 & 40.60 & 75.00 & 65.20 & 77.60 & 37.59 & 75.00 & 65.20 & 94.40 & 86.45 & 93.75 & 91.30 \\
\hline Energy & 71.60 & 35.00 & 68.20 & 45.80 & 71.60 & 32.65 & 68.20 & 45.80 & 92.90 & 85.13 & 92.05 & 86.45 \\
\hline Homogeneity & 65.00 & 24.00 & 63.00 & 56.00 & 65.00 & 19.61 & 63.00 & 56.00 & 91.25 & 85.61 & 90.75 & 89.00 \\
\hline DWT & 85.00 & 30.40 & 88.20 & 81.20 & 85.00 & 29.01 & 88.20 & 81.20 & 96.25 & 83.56 & 97.05 & 95.30 \\
\hline $\begin{array}{c}\text { Hybridization } \\
\text { without using } \\
\text { DWT }\end{array}$ & 91.60 & 73.20 & 95.20 & 84.20 & 91.60 & 57.91 & 95.20 & 84.20 & 97.90 & 95.09 & 98.80 & 96.05 \\
\hline $\begin{array}{c}\text { Hybridization } \\
\text { using DWT }\end{array}$ & 86.20 & 77.80 & $\mathbf{9 6 . 4 0}$ & 88.20 & 86.20 & 62.74 & $\mathbf{9 6 . 4 0}$ & 88.20 & 96.55 & 95.98 & $\mathbf{9 9 . 1 0}$ & 97.05 \\
\hline
\end{tabular}

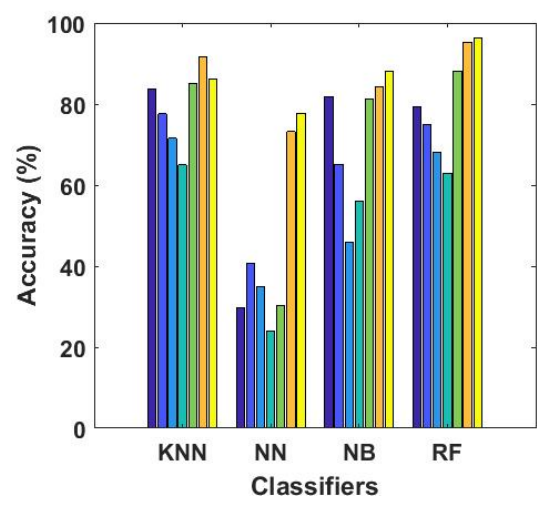

\begin{tabular}{|ll|}
\hline & Contrast \\
\hline & Correlation \\
\hline & Energy \\
\hline & Homogeneity \\
\hline & DWT \\
\hline & Hybridization without using DWT \\
\hline$\square$ & Hybridization using DWT \\
\hline
\end{tabular}

Figure.6 Accuracy comparison of proposed and existing techniques with $80 \%$ of noise

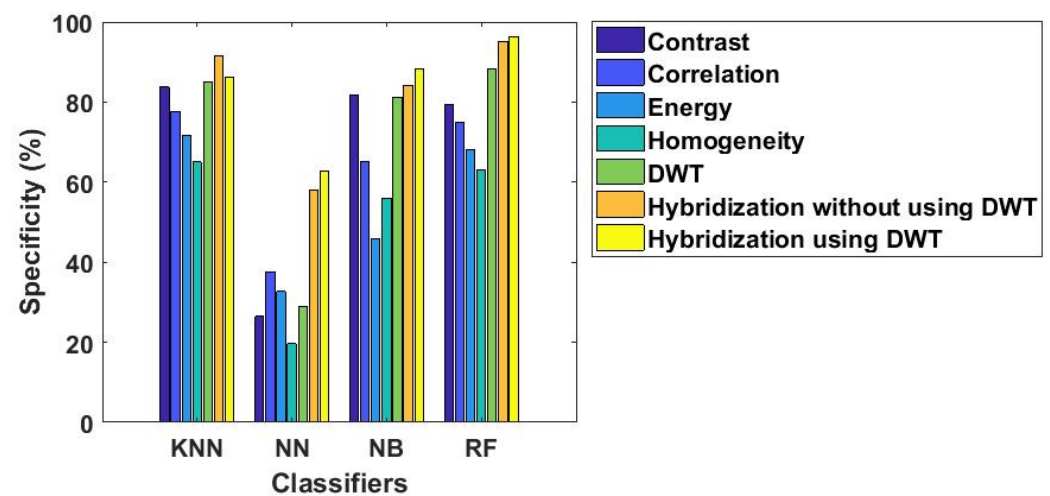

Figure.7 Specificity comparison of proposed and existing techniques with $80 \%$ of noise

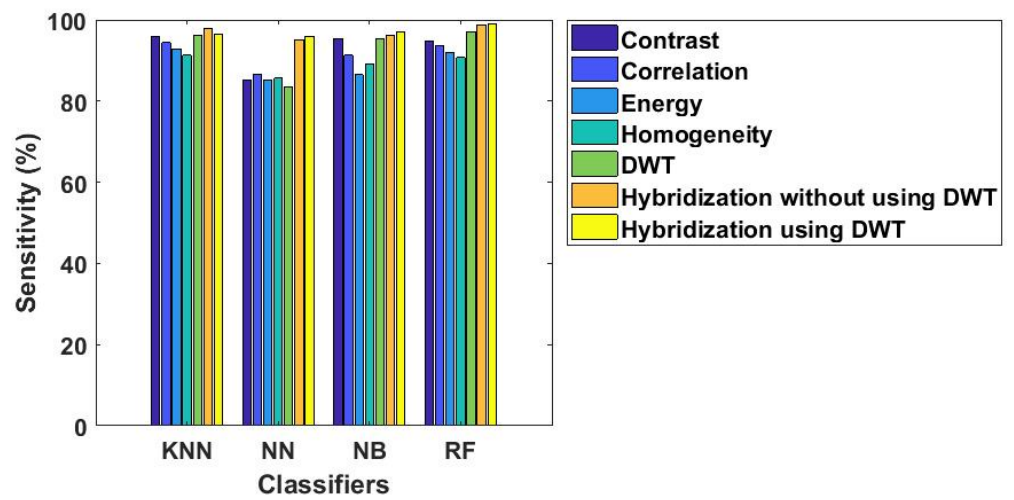

Figure. 8 Sensitivity comparison of proposed and existing techniques with $80 \%$ of noise 


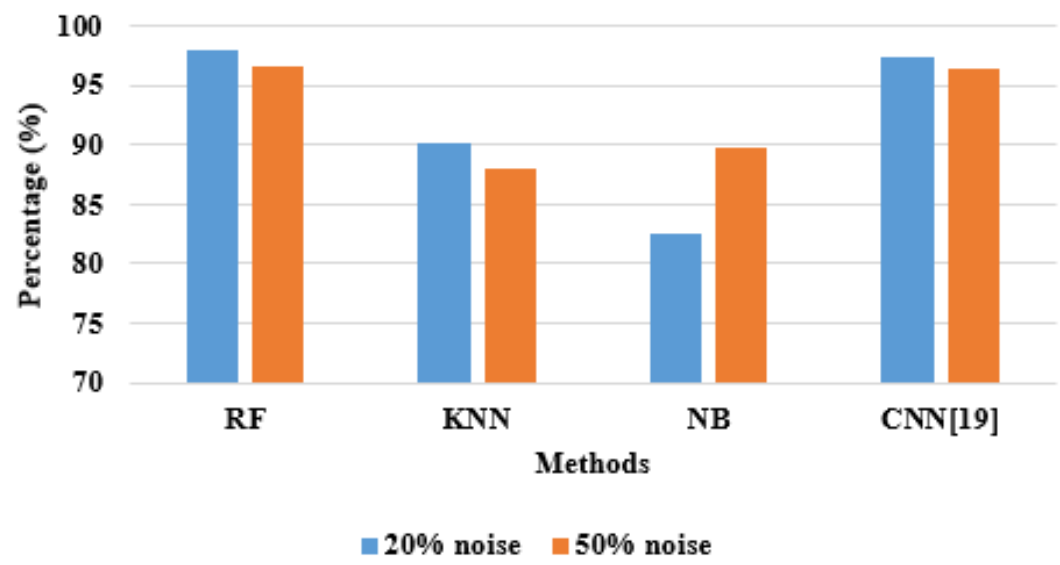

Figure.9 Accuracy comparison of proposed and existing methodologies

Table 3. Accuracy comparison of proposed and existing methodologies

\begin{tabular}{|c|c|c|c|c|}
\hline \multicolumn{5}{|c|}{ Prediction accuracy (\%) } \\
\hline Noise percentage & RF & KNN & NB & CNN [20] \\
\hline $20 \%$ & 97.90 & 90.09 & 82.60 & 97.40 \\
\hline $50 \%$ & 96.67 & 88 & 89.70 & 96.34 \\
\hline
\end{tabular}

Table 4. Suggest the threshold value for image noise

\begin{tabular}{|c|c|c|c|c|c|c|}
\hline $\begin{array}{c}\text { Contrast } \\
\text { threshold range }\end{array}$ & $\begin{array}{c}\text { Correlation } \\
\text { threshold } \\
\text { range }\end{array}$ & $\begin{array}{c}\text { Energy } \\
\text { threshold } \\
\text { value }\end{array}$ & $\begin{array}{c}\text { Homogeneity } \\
\text { threshold } \\
\text { value }\end{array}$ & $\begin{array}{c}\text { DWT } \\
\text { threshold } \\
\text { value }\end{array}$ & $\begin{array}{c}\text { Noise in } \\
\text { image }\end{array}$ & $\begin{array}{c}\text { Suitable } \\
\text { filtering }\end{array}$ \\
\hline $8.7389-9.7739$ & $0.2042-0.2162$ & $0.2199-0.2386$ & $0.6573-0.6612$ & $125.92-131.3$ & $\begin{array}{c}\text { Salt and } \\
\text { pepper } \\
\text { noise }\end{array}$ & Median filter \\
\hline $1.5929-1.8494$ & $0.1592-0.1801$ & $0.3630-0.3894$ & $0.7591-0.7808$ & $47.39-51.93$ & $\begin{array}{c}\text { Speckle } \\
\text { noise }\end{array}$ & $\begin{array}{c}\text { Mean } \\
\text { convolution) } \\
\text { filter }\end{array}$ \\
\hline $0.5144-0.5322$ & $0.9108-0.9317$ & $0.1993-0.2163$ & $0.8617-0.8630$ & $249.6-260.37$ & $\begin{array}{c}\text { Gaussian } \\
\text { noise }\end{array}$ & Gaussian filter \\
\hline $4.8863-5.2895$ & $0.0999-0.1057$ & $0.2715-0.2906$ & $0.6740-0.6782$ & $76.352-77.71$ & $\begin{array}{c}\text { Local } \\
\text { variance } \\
\text { noise }\end{array}$ & Adaptive filter \\
\hline $0.0053-0.0061$ & $0.0054-0.0062$ & $0.9874-0.9894$ & $0.9970-0.9973$ & $8.063-8.3607$ & $\begin{array}{c}\text { Poisson } \\
\text { noise }\end{array}$ & Wiener filter \\
\hline
\end{tabular}

Table 3 determines the predicted accuracy of proposed and existing methodologies with different noise ranges: $20 \%$ and $50 \%$ (minimum noise density to maximum noise density). The table 3 , shows that the classifier (RF) outperformed the existing classifiers like KNN, NB and CNN [20] for all the different noise ranges. Compared to other classifiers, $\mathrm{RF}$ is good at dealing with outliers in training data, which determines ancillary information like variable importance and classification error, easy to parameterize, non-parametric, and capable of using categorical and continuous datasets. The proposed methodology shows $5-10 \%$ of improvement in noise prediction accuracy compared to the existing methodologies. The graphical representation of accuracy comparison is determined in the Fig. 9.

\subsection{Recommending denoising algorithms}

This section determines the type of noise and suitable denoising algorithm for obtained feature or threshold values. Here, the threshold value is obtained for 20 random sample images with different noise ranges. These extracted threshold values are changed based on the number of iterations. In this scenario, the result is obtained for 50 number of iterations, which is denoted in the table 4. In case, if the noise range is increased, the threshold value of contrast, correlation and DWT is inversely proportional to the noise range. Similarly, if the noise range is increased, the threshold value of energy and homogeneity is directly proportional to the noise range. 


\section{Conclusion}

This paper evaluated a statistical investigation of noise detection that was conducted on the digital images. The scope of this experiment was to identify the noise in the corrupted image and also recommend the denoising algorithm for the respective predicted noise. In this scenario, texture feature extraction with two dimensional-DWT is implemented for extracting the optimal feature values. The extracted feature values are classified by using the classifier: RF. Associated with the other obtainable approaches in noise prediction, the proposed scheme delivered an effective performance by means of accuracy, sensitivity and specificity, around $5-20 \%$ enhancement than the previous methods. In the future work, for further improving the noise prediction rate, the descriptor level features are combined with an appropriate multiobjective classifier.

\section{References}

[1] J. Kadam, K. Thakur, and A. Sapkal, "Noise Prediction Using Rough Set Theory", In: Proc. of International Conf. On Devices, Circuits and Communications, pp.1-4, 2014.

[2] K.A. Rashmi and A. Kusagur, "An improved fast edge detection for medical image based on fuzzy techniques", Fuzzy Systems, Vol.4, No.4, pp.147-150, 2012.

[3] A. M. Elmogy, E. Mahmoud, and F. A. Turki, "Image noise Detection and Removal based on Enhanced GridLOF Algorithm", International Journal of Advanced Computer Science and Applications, Vol.8, No.12, pp.454-462, 2017.

[4] G. Hanji and M. Latte, "A new impulse noise detection and filtering algorithm", Image Processing \& Communications, Vol.16, No.1-2, pp.43-48, 2011.

[5] B. Shinde, D. Mhaske, and A.R. Dani, "Study of noise detection and noise removal techniques in medical images", International Journal of Image, Graphics and Signal Processing, Vol.4, No.2, pp.51, 2012.

[6] M.H. Nezhad, and A. Azadi, "Noise Detection and Reduction Using Algorithms Based on Particle Swarm Optimization and Fuzzy Decision making", Journal of mathematics and computer science, Vol.9, No.4, pp.370-380, 2014.

[7] M. Granados, T.O. Aydin, J.R. Tena, J.F. Lalonde, and C. Theobalt, "HDR image noise estimation for denoising tone mapped images",
In: Proc. of the $12^{\text {th }}$ International European Conf. on Visual Media Production, p.7, 2015.

[8] A. Singh, and K. Nayak, "Analysis of Image Noise Removal Methodologies for High Density Impulse Noise", International Journal of Computer Science and Mobile Computing, Vol.3, No.6, pp.659-665, 2014.

[9] M. Srivastava, C.L. Anderson, and J.H. Freed, "A new wavelet denoising method for selecting decomposition levels and noise thresholds", IEEE Access, Vol.4, pp.3862-3877, 2016.

[10] T. Kirti, K. Jitendra, and S. Ashok, "Poisson noise reduction from X-ray images by region classification and response median filtering", Sādhanā, Vol.42, No.6, pp.855-863, 2017.

[11] N.J. Harish, B.B.S. Kumar, and A. Kusagur, "Hybrid robust watermarking techniques based on DWT, DCT, and SVD", International Journal of Advanced Electrical and Electronics Engineering, Vol.2, No.5, pp.137-143, 2013.

[12] B. Blaysat, M. Grediac, and F. Sur, "On noise prediction in maps obtained with global DIC", In: Proc. of International Conf. on Advancement of Optical Methods in Experimental Mechanics, Cham, Vol.3, pp.211-216. 2016.

[13] G.A. Kumar and A. Kusagur, "Evaluation of image denoising techniques a performance perspective", In: Proc. of International Conf. On Signal Processing, Communication, Power and Embedded System, pp.1836-1839, 2016.

[14] P. Fu, C. Li, W. Cai, and Q. Sun, "A spatially cohesive super pixel model for image noise level estimation", Neurocomputing, Vol.266, pp.420-432, 2017.

[15] J. Constantin, A. Bigand, I. Constantin, and D. Hamad, "Image noise detection in global illumination methods based on FRVM", Neurocomputing, Vol.164, pp.82-95, 2015.

[16] K. Ray, "Unsupervised edge detection and noise detection from a single image", Pattern Recognition, Vol.46, No.8, pp.2067-2077, 2013.

[17] C. Catal, O. Alan, and K. Balkan, "Class noise detection based on software metrics and ROC curves", Information Sciences, Vol.181, No.21, pp.4867-4877, 2011.

[18] X. Tian and E. Samei, "Accurate assessment and prediction of noise in clinical CT images", Medical physics, Vol.43, No.1, pp.475-482, 2016.

[19] J. Joseph and R. Subban, "A Novel Denoising Algorithm Based on Superpixel Clustering and Dictionary Learning Approach", International 
Journal of Intelligent Engineering and Systems, Vol.11, No.1, pp.142-152, 2018.

[20] S.S. Roy, M. Ahmed, and M.A.H. Akhand, "Classification of massive noisy image using auto-encoders and convolutional neural network", In: Proc. of the $8^{\text {th }}$ International Conf. On Information Technology, pp. 971-979, 2017. 